\title{
Design and Implementation of Hybrid Energy Harvesting System for Low Power Devices
}

\author{
Akshaj Arora, Sahitya Singh, Neel Chatterjee, Malay RanjanTripathy and Sujata Pandey* \\ Amity University Uttar Pradesh, Noida - 201313, Uttar Pradesh, India; akshajarora1@gmail.com, \\ sahitya.singh25@gmail.com,spandey@amity.edu,mrtripathy@amity.edu
}

\begin{abstract}
Objectives: In this paper we report on experimental studies and system design for harnessing energy from three alternate energy sources, which are solar energy, radio frequency energy and piezoelectric energy. Methods/Statistical Analysis: In order to make an integrated unit, we have made a case like structure of the device. For RF energy harvesting, we have designed two antennas on Ansoft HFSS and then got them fabricated. For RF to DC power conversion we have used schottky detectors which are capable of highly efficient impedance matching. Moving on to solar energy, we have used two solar panels and each can produce voltage up to five volts. The panels are placed both inside and outside the system, to ensure that we get power when case is open as well as when it is closed. Finally, for the piezoelectric energy harvesting, we have made a series and parallel combination of piezoelectric sensors and this network is followed by a voltage doubler circuit, which is used to augment the voltage produced by the combination of sensors. Findings: Though the system was designed for harvesting solar as well as piezo energy, our main focus was to harvest energy from RF energy. Power is harvested from both the antennas and it is shown that lotus shaped patch antenna is giving more DC voltage $44 \mathrm{mV}$ and $63 \mathrm{mV}$ at $900 \mathrm{MHz}$, $1800 \mathrm{MHz}$ respectively at $50 \mathrm{~cm}$ than Multi patch fractal antenna is giving maximum voltage $60 \mathrm{mV}, 24 \mathrm{mV}$ and $34 \mathrm{mV}$ at $900 \mathrm{MHz}, 1800 \mathrm{MHz}$ and $2400 \mathrm{MHz}$ respectively at $50 \mathrm{~cm}$. Application/Improvements: Energy has been successfully harnessed from all three energy sources i.e. RF energy, piezoelectric, solar energy. The system can be used to power low power devices.
\end{abstract}

Keywords: Antenna, Energy Harvesting, RF Energy, Piezoelectric, SolarSensor Networks (WSNs)

\section{Introduction}

The augmenting consumption of energy has resulted in our dependency on fossil fuels such as coal, oil and gas. These conventional sources of energy exhibit pernicious effects not only on environment but also on the existing biodiversity. Also, these sources of energy are likely to be exhausted in the coming future. Therefore, researchers and scientists are working hard in order to harness energy from various alternate sources like solar, wind, geothermal etc. ${ }^{1-10}$. Energy harvesting is a process of harvesting power from ambient energy. Generally energy harvesting technique and system concentrate on renewable sources. Large solar panels and wind turbines have become one of the major source of energy harvesting in sunny and windy region cause of it abundant availability. Even water resources are being used in energy harvesting. Many hydroelectric power generators are established near dams and water bodies. Researchers have done these complex conversion in innovative way were presented $11-17$,

Unlike renewable resources, another alternate source of energy harvesting is wireless power transmission through microwave signal or electromagnetic wave $e^{11,13,14,16-1}$, The demand of wireless sensor networks has exponentially augmented in various fields such as medicals systems, industrial networking, military applications, smart grid, pollution monitoring and so on. The wireless sensor requires energy in communication, sensing and storage of sensed data. Earlier also researchers have build hybrid energy harvesting system using different alternative energy. For instances, US patent US 7268517 B2,comprises of RF energy Collector, solar energy

*Author for correspondence 
collector and vibrational energy collector along with power storage for storing electrical power wherein array of antenna is being used for harvesting electromagnetic energy and electronic circuits for harvesting mechanical energy. In Patent US 20070216348 A1, antenna circuit and antenna booster is designed to harvest radio signals for generating power. One such alternate energy genesis is "Radio Frequency" In order to use solar electricity practically i.e. in daily used appliances, we require a voltage or current for their operation, for which a number of solar cells are connected together to form a solar panel, also called a PV module 12,16 . Also piezo energy harvesting, it has been investigated ${ }^{2,13}$ Most piezoelectric electricity sources produce power of the order of milliwatts

In this paper we report on the development of a hybrid energy harvesting system. In order to make an integrated unit, we have made a case like structure of the device. For RF energy harvesting, we have designed two antennas on Ansoft HFSS and then got them fabricated. For RF to DC power conversion we have used schottky detectors which are capable of highly efficient impedance matching. Moving on to solar energy, we have used two solar panels and each can produce voltage up to five volts. The panels are placed both inside and outside the system, to ensure that we get power when case is open as well as when it is closed. Finally, for the piezoelectric energy harvesting, we have made a series and parallel combination of piezoelectric sensors and this network is followed by a voltage doubler circuit, which is used to augment the voltage produced by the combination of sensors.

\section{System Design}

The system consists of integration of all the above mentioned energy harvesting technique that is RF energy, solar energy and Piezo electric energy harvesting together in a single system. The proposed system is designed in such a way that it will harvest energy even if there is only one source is available. The design of the device is taken as case like structure so that it will be easier to carry it or making it a portable device. The arrangement of the components is done according to the efficient use of availability of sources. Both the antennas are kept inside the box and piezo is used on the areas which will be used for holding the box. In this case, piezo is used on top of the box and two solar panels are used and each produces maximum $5 \mathrm{~V}$, one place on the top side of box and one inside the box. This arrangement is done to make the system more reliable and versatile. In all the cases, whether system is open or closed, kept or held, it will be harvesting energy from ambient or available sources. For instance, if the system is closed or kept, the solar panel and piezo will harvest energy and if the system is open then antenna and solar panel will harvest the energy. Figure 1 (a) shows the top view of the system and (b) shows the inside view.

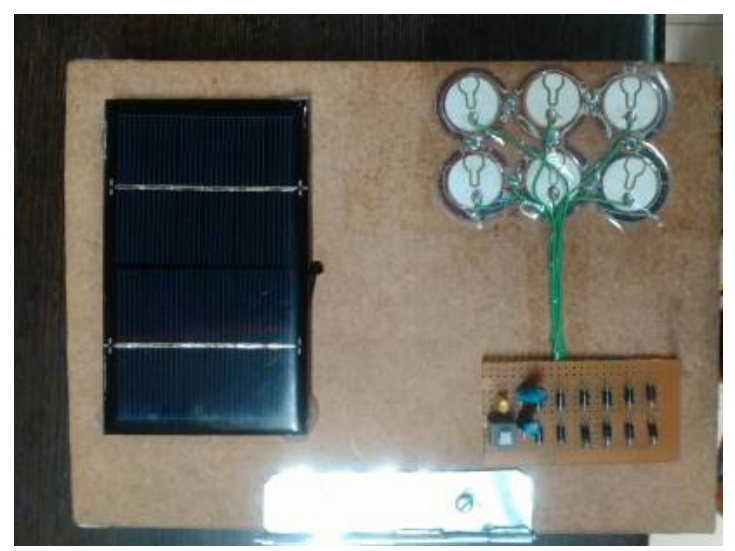

(a)

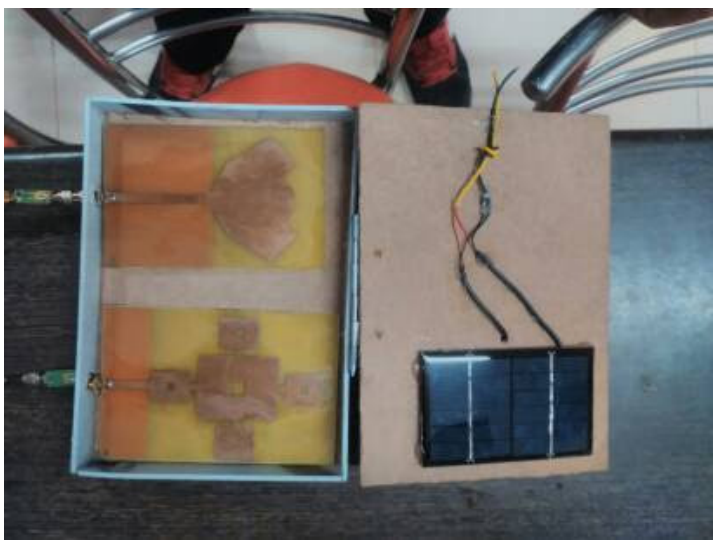

(b)

Figure 1. (a) Closed view of proposed prototype (b) Open view of proposed prototype.

\section{Experimentation}

An antenna, matching and rectifying circuits are the basic building blocks of a RF energy harvesting system. Each block of the energy harvesting system plays an important role. Figure 2 shows the block diagram of the RF energy harvesting system. 
To show RF energy harvesting, a setup is done using a transmitting antenna, receiving antenna (designed antenna), tripod stand, trans-receiver device of range 0.04 $\mathrm{GHz}$ to $4.4 \mathrm{GHz}, \mathrm{RG} 141$ connectors and scale.

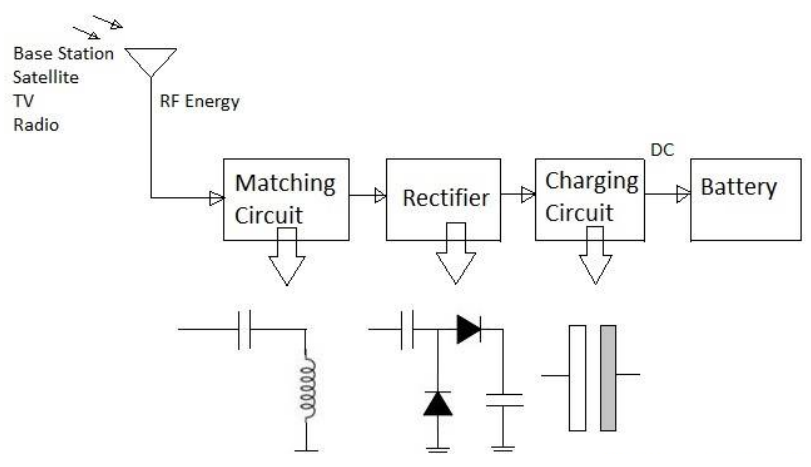

Figure 2. Block diagram of RF energy harvesting.

Figure 3 shows the experimental setup done for measuring the harvested RF energy.

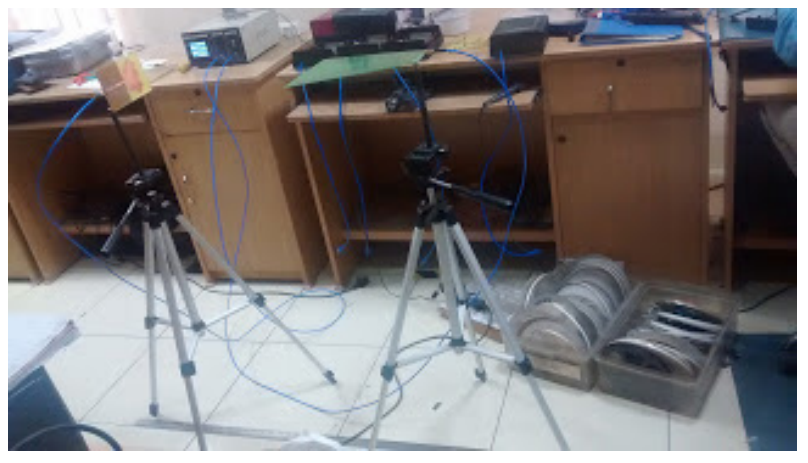

Figure 3. Setup for RF energy harvesting.

The transmitting antenna used in this measurement is planner horn shaped antenna. To measure the power received by the designed antenna, transmitting antenna and designed antenna is connected to the antenna training device via RG141 wire at the output and input port respectively. Power of $+10 \mathrm{dBm}$ is transmitted through the planner horn shaped antenna.

Keeping in mind our target frequencies that is, $900 \mathrm{MHz}, 1800 \mathrm{MHz}, 2400 \mathrm{MHz}, 3500 \mathrm{MHz}$ and 5800 $\mathrm{MHz}$, we have tuned our testing antenna and measured the power received at each frequency respectively. The distance between the transmitting antenna and receiving antenna is initially kept $50 \mathrm{~cm}$, because it is essential to keep the receiving antenna in the far field region of the source antenna. Since antenna radiates spherical wave and at far field region the spherical wave front approximately become the planar wave. The inverse square law of power that is, power is inversely proportional to the square of distance, is demonstrated by increasing the distance between the receiving and transmitting antenna. The distance between the two antennas is varied from $50 \mathrm{~cm}$ to $100 \mathrm{~cm}$ with an interval of $10 \mathrm{~cm}$.

After designing an efficient antenna with significant return loss, radiation pattern, gain etc, which can receive power from source antenna, other challenge that is faced by researcher is in designing a matching circuit. The efficiency of rectifier degraded is the operating frequency changed from resonance frequency of the rectifier. As we know that the filter functionality is to match the antenna impedance and the conjugate impedance of rectifier input. Also, the rectifier input varies as a function of frequency and incident power and antenna impedance may also vary with frequency. Therefore, impedance matching is one of the crucial elements of RF energy harvesting. Mismatching in impedance decreases the RF-DC conversion efficiency. In case of multiband frequency, it is necessary to design individual rectifier circuits for each frequency so that the rectenna can harvest energy from each operating frequency. For this reason, we have used an RF to DC detector that is Agilent 33330c, to measure the DC voltage output converted from the received RF power from designed antenna. This device efficaciously maintains $50 \mathrm{ohm}$ impedance matching over a frequency range of $0.01 \mathrm{GHz}$ to $26.5 \mathrm{GHz}$. The setup for measuring DC voltage remained the same except, in this case we have connected the rectifier to the designed antenna and measured the DC voltage through millimeter. Similar to the previous experiment, the distance between the two antennas is varied from $50 \mathrm{~cm}$ to $100 \mathrm{~cm}$ with an interval of $10 \mathrm{~cm}$.

Figure 4 (a), (b) and (c) shows the experimental setup being done to measure the output power from two antennas being designed.

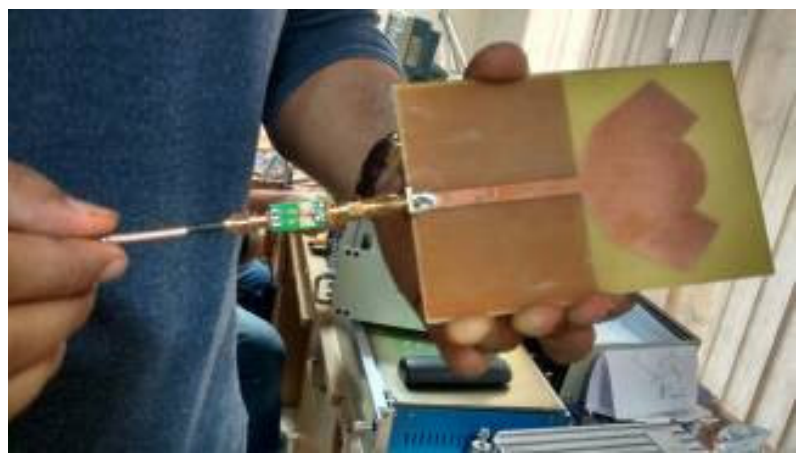

(a) 


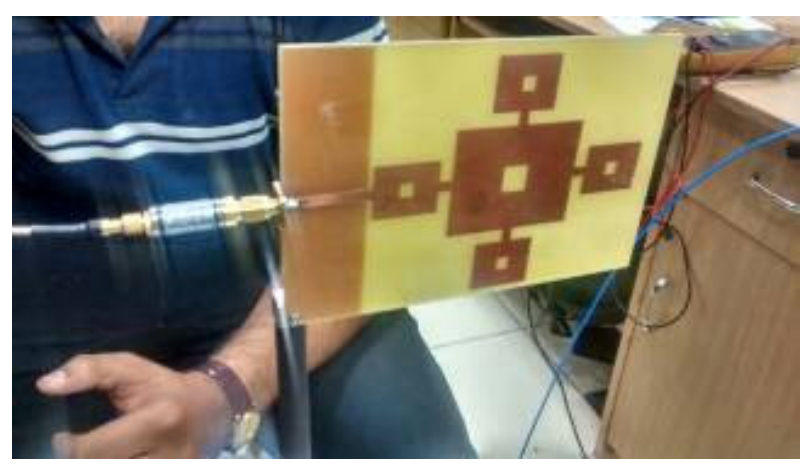

(b)

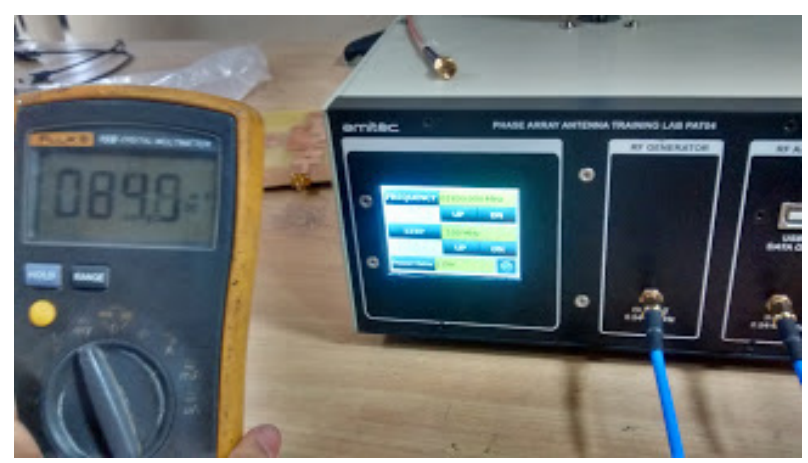

(c)

Figure 4. (a) Lotus shaped patch antenna with RF-DC detector (b) Multi-Patch Fractal antenna with RF to DC detector that is Agilent 33330C (c) Measured power and DC voltage received by antenna.

In this paper, along with RF energy harvesting and solar energy harvesting, energy is also harvested by using piezoelectric. These sensors convert mechanical energy that is, the force applied to electrical energy, these sensors are made up of quartz. Since only few millivolts of voltage are generated from a single sensor, a series and parallel combination of piezoelectric sensors is made to generate more power. The diode used in the circuit is IN4007. The circuitry is illustrated in the figure 5. Still after making a network of these sensors, only few hundred millivolts were generated when force was applied, which is not even enough for lighting up a led. In order to further augment the voltage, a voltage doubler circuit is used. Basically, this circuit will convert AC outputs of each sensor to DC and all voltages will be added up. Two capacitors are used to store charge for some time. When the switch is on the output of the circuit is connected to the led, which acts like a load for the circuit and it is visible that the LED glows. When the switch is off, there is no connection between the circuit and LED, the capacitors get charged and the maximum measured output of the circuit is 11 volts.
As already mentioned the usage of two solar cell in the system, we have connected diode for parallel connection so that according to the box, one of the solar panel will work efficiently.

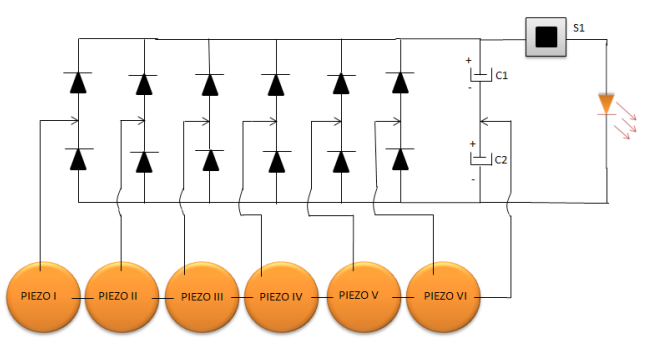

Figure 5. Circuitry for piezo energy harvesting.

\section{Discussion of Results}

Though the system was designed for harvesting solar as well as piezo energy, our main focus was to harvest energy from RF energy. Figure 6 (a) and (b) show how the two antennae designed by us produce $\mathrm{dc}$ voltage and further how this voltage ca be used for powering low power devices.

Power is harvested from both the antennas and it is shown that lotus shaped patch antenna is giving more Dc voltage $44 \mathrm{mV}$ and $63 \mathrm{mV}$ at $900 \mathrm{MHz}, 1800 \mathrm{MHz}$ respectively at $50 \mathrm{~cm}$ than Multi patch fractal antenna is giving maximum voltage $60 \mathrm{mV}, 24 \mathrm{mV}$ and $34 \mathrm{mV}$ at 900 $\mathrm{MHz}, 1800 \mathrm{MHz}$ and $2400 \mathrm{MHz}$ respectively at $50 \mathrm{~cm}$.

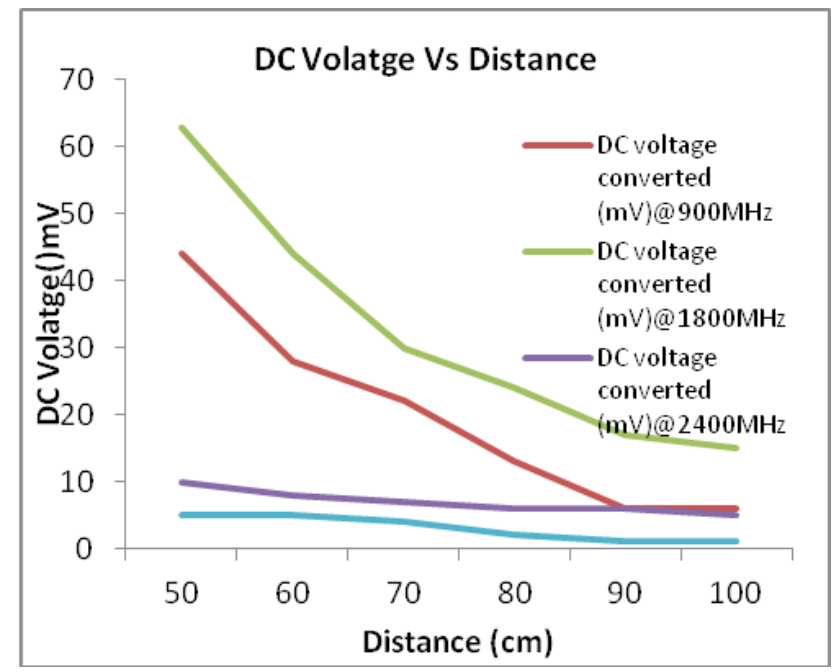

(a) 


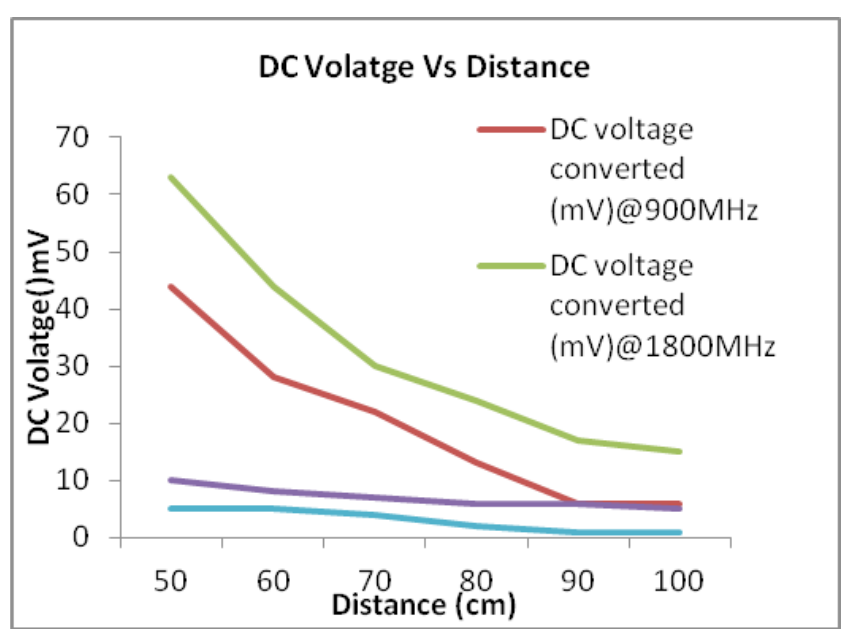

(b)

Figure 6. (a) Power vs. distance graph of multi patch fractal antenna (b) Power vs. distance graph of lotus shaped patch antenna.

\section{Conclusion}

Energy has been successfully harnessed from all three energy sources i.e. RF energy, piezo electric, solar energy. It was observed that the maximum DC power obtained after scavenging RF energy and converting it into DC is 51 micro watts, which can be used for powering a watch, calculator, passive RFID, $32 \mathrm{KHz}$ quartz oscillator et cetera. The main challenge faced while conducting experiments was impedance matching. Since, energy is to be harvested from various target bands, which are GSM 900, GSM 1800, IoT (2.45 GHz), Wi-Max (3.5 $\mathrm{GHz}$ ), it is very essential and an arduous task to maintain a $50 \mathrm{ohm}$ impedance throughout the target frequency range. For piezoelectric energy harvesting, a seriesparallel combination of piezoelectric sensors, which are made up of quartz is made to get a reasonable voltage. Since, an optimum output voltage was not harnessed; a voltage doubler was used to boost the voltage. Maximum measured output voltage of this system was 11 volts. For solar energy harvesting, we have used two solar panels, one outside the box and another inside it. In order to obtain a single output of the solar system, two diodes IN4007 are used to avoid back voltage. The outputs of all three systems are not joined in order to measure them separately.

\section{Acknowledgement}

The authors are thankful to AMITEC for carrying out the experiments and measurements.

\section{References}

1. Ali G, Wagner J, Moline D, Schweisinger T. Energy harvesting from atmospheric variations-theory and test. Elsevier, Renewable Energy. 2015 Feb; 74:528-35.

2. Din NM, Chakrabarty CK, Bin Ismail A, Devi KKA, Chen W-Y. Design of RF harvesting system for energizing low power devices. Progress in Electromagnetic Research. 2012; 132:49-69.

3. Naderi, Yousof M, Nintanavongsa P, Chowdhury KR. RFMAC: A medium access control protocol for re-chargeable sensor networks powered by wireless energy harvesting. IEEE Transactions on Wireless Communications. 2014; 13(7):3926-37.

4. Mishra D, Swades D, Chowdhury KR. Charging time characterization for wireless RF energy transfer. IEEE Transactions on Circuits and Systems II: Express Briefs. 2015; 62(4):362-6.

5. Xie K, et al. Harvest the ambient AM broadcast radio energy for wireless sensors. Journal of Electromagnetic Waves and Applications. 2011; 25(14-15):2054-65.

6. Mickle $\mathrm{MH}$, et al. A circuit model for passive RF autonomous devices with protocol considerations. International Journal of Computers and Applications. 2006; 28(3):24350.

7. Hossain MI, Faruque MRI, Islam MT. Low SAR microstrip patch antenna for mobile phone. Frequenz. 2015; 69(910):399-405.

8. Thierry T, Ludivine F, Laurent O, Romain B, Florent T, Valerie V. RF energy harvesting and remote powering at 900 $\mathrm{Mhz}$ and 2.4 GHz. IEEE International Conference on Electronics, Circuits and Systems (ICECS); 2014 Dec.

9. Pinuela M, Mitcheson PD, Lucyszyn S. Ambient RF energy harvesting in urban and semi-urban environments. IEEE Transactions on Microwave Theory and Techniques. 2013 Jul; 61(7):2715-26.

10. Nintanavongas P. A survey on RF energy harvesting: circuits and protocols. Elsevier, Energy Procedia. 2014; 56:412-22.

11. Shariati N, Rowe WST, Scott JR, Ghorbani K. Multi-service highly sensitive rectifier for enhanced RF energy scavenging. Sci Rep. 2015; 5:9655.

12. Gambier P, Anton SR, Kong N, Erturk A, Inman DJ. Piezoelectric, solar and thermal energy harvesting for hybrid low-power generator systems with thin-film batteries. Meas Sci Technol. 2012; 23:015101.

13. Roundy S, Wright PK. A piezoelectric vibration based generator for wireless electronics. Smart Mater Struct. 2004; 13:1131-44. 
14. Rao S, Mehta NB. Hybrid energy harvesting wireless systems: performance evaluation and benchmarking. IEEE Trans on Wireless Communications. 2014; 13(9):4782.

15. Reilly E, Miller L, Fain R, Wright PK. A study of ambient vibrations for piezoelectric energy conversion. Proceedings of the Power MEMS. Washington, DC, USA. 2009 Dec 1-4. p. 312-5.

16. Li Y, Shi R. An intelligent solar energy-harvesting system for wireless sensor networks. EURASIP Journal on Wireless Communications and Networking. 2015; 179.
17. Raghunathan V, Kansal A, Hsu J, Friedman J, Srivastava M. Design considerations for solar energy harvesting wireless embedded systems. 4th IEEE/ACM International Conference on Information Processing in Sensor Networks; 2005 Apr. 